\title{
Anti-hepatocellular carcinoma properties of the anti-alcoholism drug disulfiram discovered to enzymatically inhibit the AMPK- related kinase SNARK in vitro
}

\author{
Kaku Goto ${ }^{1}$, Naoya Kato', Raymond T. Chung ${ }^{2}$ \\ ${ }^{1}$ The Advanced Clinical Research Center, The Institute of Medical Science, The University of Tokyo, Tokyo 108-8639, Japan \\ ${ }^{2}$ Liver Center and Gastrointestinal Division, Department of Medicine, Massachusetts General Hospital, Harvard Medical \\ School, Boston, MA 02114, USA \\ Correspondence to: Raymond T. Chung, email: Chung.Raymond@mgh.harvard.edu \\ Naoya Kato, email: kato-2im@ims.u-tokyo.ac.jp \\ Keywords: disulfiram, Antabuse, SNARK, NUAK2, HCC \\ Received: April 19, $2016 \quad$ Accepted: August 24, $2016 \quad$ Published: September 02, 2016
}

\section{ABSTRACT}

We recently described that the anti-apoptotic AMPK-related kinase, SNARK, promotes transforming growth factor (TGF)- $\beta$ signaling in hepatocellular carcinoma (HCC) cells, as a potentially new therapeutic target. Here we explored FDA-approved drugs inhibiting the enzymatic activity of SNARK, using an in vitro luminescence kinase assay system. Interestingly, the long-used anti-alcoholism drug disulfiram (DSF), also known as Antabuse, emerged as the top hit. Enzymatic kinetics analyses revealed that DSF inhibited SNARK kinase activity in a noncompetitive manner to ATP or phosphosubstrates. Comparative in vitro analyses of DSF analogs indicated the significance of the disulfide bond-based molecular integrity for the kinase inhibition. DSF suppressed SNARK-promoted TGF- $\beta$ signaling and demonstrated anti-HCC effects. The chemical and enzymatic findings herein reveal novel pharmacological effects of and use for DSF and its derivatives, and could be conducive to prevention and inhibition of liver fibrosis and HCC.

\section{INTRODUCTION}

Protein kinases are critically involved in nearly all cellular functions encompassing metabolism, gene expression, proliferation, motility, and death. Their dysregulation, contributes to serious diseases such as cancer, hypertension, Parkinson's disease, and autoimmune diseases [1], which are attractive therapeutic targets [2]. In particular, AMP-activated protein kinase (AMPK) has been recognized as a key regulator of energy balance, related to type 2 diabetes and cancer [3], and AMPK-related kinases (ARKs) are also being increasingly recognized as modulators of cell dynamics and metabolism and observed to be tumor promoters though their physiological properties are not yet well understood [4].

Liver diseases including fibrosis and consequential hepatocellular carcinoma (HCC) remain an important health menace [5], in urgent need of improved methods for prophylactic and therapeutic management [6]. We recently reported that sucrose-non-fermenting protein kinase 1 (SNF1)/AMPK-related protein kinase
(SNARK), the fourth member of 14 ARKs [7], supported replication of hepatitis $\mathrm{C}$ virus (HCV), a causative agent of HCC, in genome-wide RNAi screen [8], and enhanced profibrogenic transforming growth factor (TGF)- $\beta$ signaling in HCC cells [9], suggesting SNARK as an attractive pharmacological target. Hence we here sought to find repositionable approved drugs [10] inhibiting SNARK kinase activity implementing an in vitro luminescence kinase assay system. Interestingly, the top hit in an FDA-approved drug library was disulfiram (DSF), the long-used anti-alcoholism drug also known as Antabuse. Enzymatic kinetics analyses revealed that DSF inhibited SNARK kinase activity in a noncompetitive manner to ATP or phosphosubstrates. While a close DSF analog tetramethylthiuram disulfide (TR) exerted inhibition, its monosulfide analog tetramethylthiuram monosulfide (TMTM) did not, indicating the significance of the disulfide bond-based molecular integrity of DSF for the kinase inhibition. DSF was indeed proved in cell culture to suppress SNARK-enhanced TGF- $\beta$ signaling monitored through plasminogen activator inhibitor (PAI)-1 activity. DSF also exhibited anti-HCC effects, to which HCC cells 
were conferred resistance by excessive supply of SNARK. The successful discovery of a SNARK inhibitor here discloses a novel mode to explain the recently recognized anti-cancer effects and anti-fibrogenic potential of DSF $[11,12]$. These chemical and enzymatic findings will be conducive to prevention and inhibition of liver fibrosis and HCC.

\section{RESULTS}

\section{Screen for SNARK kinase inhibitors in vitro}

To confirm the validity of the in vitro SNARK kinase assay system, which reports the amount of ATP consumed for phosphorylation as luminescent signals, we first tested the effects of staurosporine (STS), a known multi-protein kinase inhibitor [13], and consequently observed significantly decreased luminescence by STS (Figure 1A), authenticating the monitoring method. Next we implemented the kinase assay for 636 compounds in the FDA-Approved Drug Screen-well Library (Figure 1B); only three compounds demonstrated inhibition more than $90 \%$ in the primary screen and the top hit with around 97\% inhibition was DSF, the long-used anti-alcoholism drug also known as Antabuse (Figure 1C). DSF was structurally independent from other hits with inhibition more than 50\% (Supplementary Figure S1A and S1B). The robust inhibition by DSF more potent than other highranking candidates was validated separately, with the dosedependent effects giving 50\% inhibitory concentration $\left(\mathrm{IC}_{50}\right)$ of $43.7 \mu \mathrm{M}$ in vitro (Figure $1 \mathrm{D}$ ), while luciferase activity itself was not suppressed by DSF (Supplementary Figure S1C) in agreement with the previous counterscreen [14].

\section{Kinetics and modes of SNARK kinase inhibition by DSF}

In order to clarify the mode of SNARK inhibition by DSF, enzymatic kinetics in the presence of DSF were investigated. The Michaelis-Menten plots of the kinase reaction with various concentrations of ATP (Figure 2A) implied non-competitive inhibition against ATP, which was confirmed by the Lineweaver-Burk and Dixon plots (Figure 2B and $2 \mathrm{C}, \mathrm{K}_{\mathrm{m}}=19.3 \pm 2.18$ $\left.\mu \mathrm{M}, \mathrm{K}_{\mathrm{i}}=65.7 \pm 6.09 \mu \mathrm{M}\right)$. In addition, the reaction was also examined with various concentrations of the phosphosubstrate peptide CHKtide, whose kinetics are shown in the Michaelis-Menten plots (Figure 2D), with $\mathrm{K}_{\mathrm{m}}=48.9 \pm 4.23 \mu \mathrm{M}$. The Lineweaver-Burk and Dixon plots revealed non-competitive inhibition against the phosphosubstrates (Figure $2 \mathrm{E}$ and $2 \mathrm{~F}, \mathrm{~K}_{\mathrm{i}}=64.3 \pm$ $5.17 \mu \mathrm{M}$ ), confirmed by no observation of competitive inhibition after the preincubation of the kinase with DSF either (Supplementary Figure S2).

Next, to address the question of the functional groups responsible for the inhibition, the effects of the close DSF analogs TR and TMTM (Figure 3A), on the kinase reaction were tested; in actuality TR inhibited the kinase activity with the $\mathrm{IC}_{50} 42.0 \mu \mathrm{M}$ (Figure $3 \mathrm{~B}$ ), and the Michaelis-Menten (Figure 3C), Lineweaver-Burk (Figure $3 \mathrm{D})$, and Dixon (Figure 3E) plots $\left(\mathrm{K}_{\mathrm{m}}=21.8 \pm 1.54 \mu \mathrm{M}\right.$, and $\left.\mathrm{K}_{\mathrm{i}}=79.0 \pm 5.25 \mu \mathrm{M}\right)$ suggested ATP-noncompetitive inhibition. Again kinase assays at various CHKtide concentrations produced the Michaelis-Menten (Figure 3F), Lineweaver-Burk (Figure 3G), and Dixon (Figure $3 \mathrm{H})$ plots $\left(\mathrm{K}_{\mathrm{m}}=51.2 \pm 5.00 \mu \mathrm{M}, \mathrm{K}_{\mathrm{i}}=138.0 \pm 21.3 \mu \mathrm{M}\right)$, indicating phosphosubstrate-noncompetitive inhibition. Meanwhile TMTM exhibited almost no inhibitory activities (Figure 3I) up to $1000 \mu \mathrm{M}$ (Supplementary Figure S3A) and hence the disulfide bond was exhibited in vitro to be important for DSF to inhibit the SNARK kinase activity. With respect to the DSF metabolite diethyldithiocarbamate (DDC) (Figure 3A) [15], no inhibitory effects were displayed (Figure 3J) while only slight inhibition was observed at higher concentrations than $100 \mu \mathrm{M}$ (Supplementary Figure S3B), and neither did S-Methyl-N, N-diethylthiocarbamoyl sulfoxide (DETC$\mathrm{MeSO}$ ) (Figure 3K), a further metabolite downstream of DDC and the known inhibitor of aldehyde dehydrogenase [15], inhibit the kinase activity in vitro up to $100 \mu \mathrm{M}$ (Figure 3L) with mild inhibition at higher concentrations (Supplementary Figure S3C), wherefore biochemical significance of the DSF structure was confirmed. Thus enzymatic kinetics and chemical structure analyses uncovered the ATP- and phosphosubstrate-noncompetitive modes of SNARK inhibition by DSF via disulfide bondbased molecular integrity (Supplementary Table S1).

\section{Inhibition of SNARK-promoted TGF- $\beta$ signaling by DSF}

As we recently reported that SNARK enhanced TGF- $\beta$ signaling in HCC cells and the pharmacological inhibition of SNARK resulted in the suppression of profibrogenic signaling [9], the effects of DSF were next assessed in cell culture. The activity of a plasmid PAI/L encoding a luciferase reporter gene driven by promoter sequences of PAI-1, a transcriptional target of TGF- $\beta$ [16], was enhanced by SNARK overexpression and the effects were further highlighted by TGF- $\beta$ in HepG2 cells (Figure 4A, white bars) in accordance with our previous data [9]. DSF suppressed the SNARK-promoted luciferase activities in the absence and particularly presence of TGF- $\beta$ (Figure 4A, black bars), endorsing the inhibitory efficacy in vitro, while no inhibition of luciferase activity itself by DSF was confirmed in HepG2 cells with the overexpression of luciferase protein (Supplementary Figure S4A). In turn, RNAi-mediated knockdown of SNARK expression decreased PAI/L activity whether treated with TGF- $\beta$ or not (Figure $4 \mathrm{~B}$, white bars) consistent with past report [9], and the suppressive effects by DSF on PAI/L were relatively blunted (Figure 4B, black bars), indicating the dependence of the DSF-mediated 
TGF- $\beta$ signaling inhibition on SNARK. Subsequently, the DSF analogs TR and TMTM with and without SNARK kinase inhibitory activity in vitro, respectively, were tested in the same setting. TR inhibited the SNARKenhanced PAI/L activity, demonstrating all the more pronounced effects in the presence of TGF- $\beta$ (Figure $4 C$ ), while TMTM did not affect PAI/L significantly
(Figure 4D). Intriguingly, DDC, a key metabolite of $\mathrm{DSF}$, decreased the PAI/L activity as well in the presence of TGF- $\beta$ (Figure 4E) unlike DETC-MeSO (Figure 4F) though the both lacked the kinase inhibitory properties in vitro (Figure 3J and 3L). Therefore, possibly responsible factors such as other DDC metabolites than DETC$\mathrm{MeSO}$ and/or intracellular biological effects of DDC
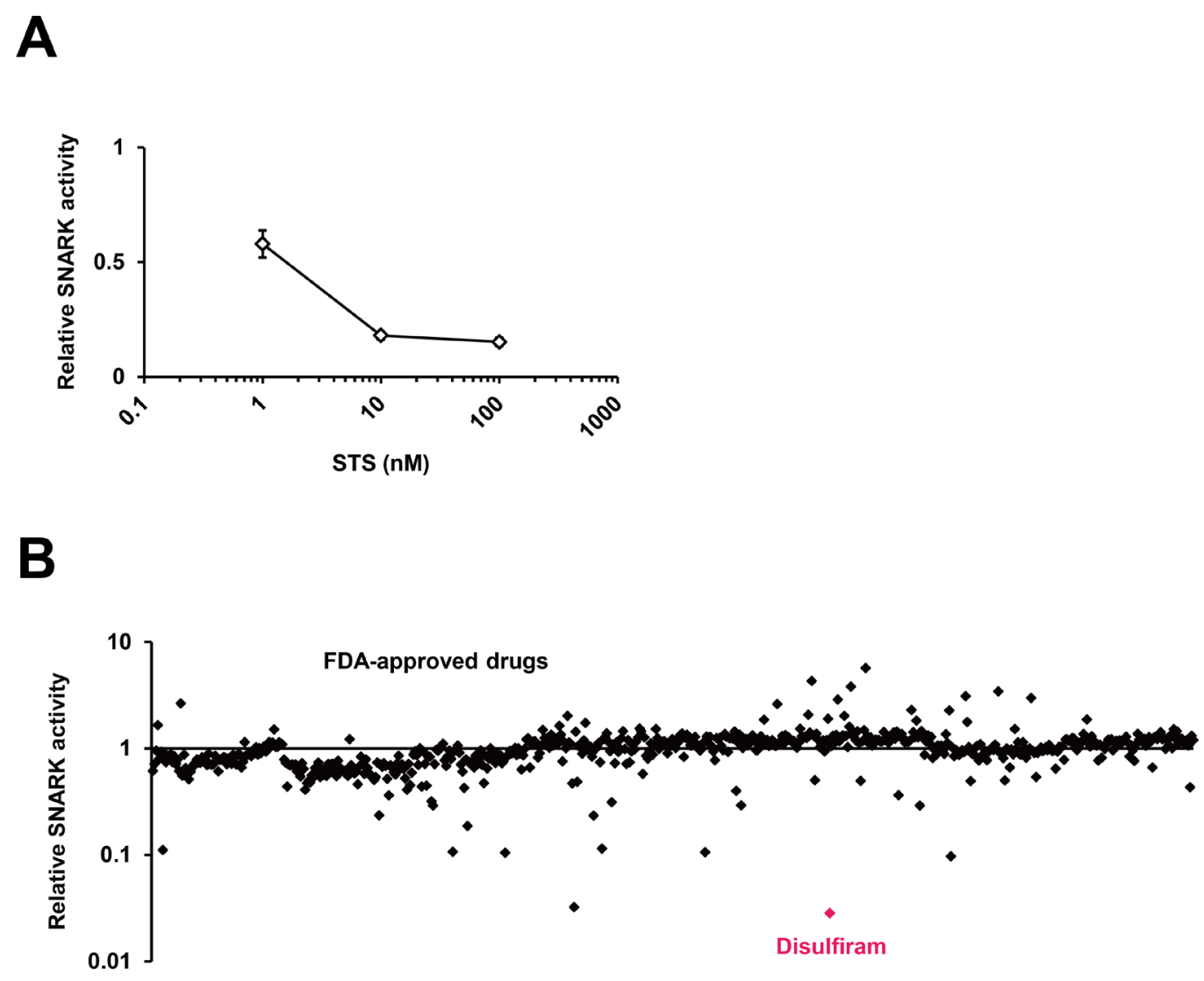

C<smiles>CCN(CC)C(=S)SSC(=S)N(CC)CC</smiles>

Disulfiram

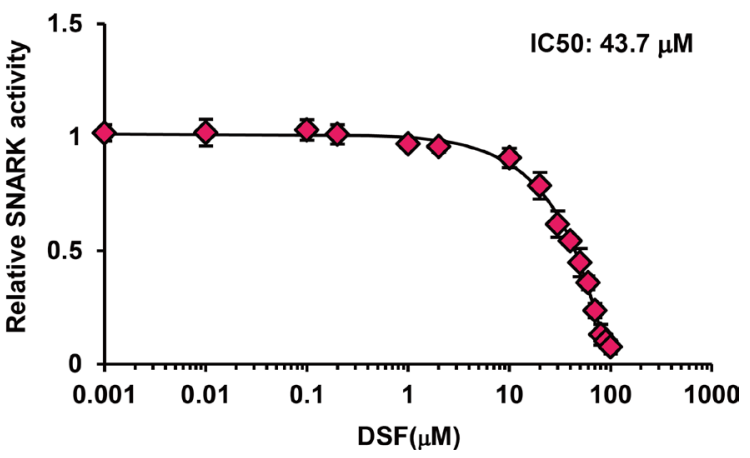

Figure 1: The SNARK kinase inhibitor screen in vitro. A. The in vitro SNARK kinase assay system was validated using the known multi-kinase inhibitor STS as the control. B. The kinase assay was performed for 636 compounds in the FDA-Approved Drug Screen-well Library. Z scores of relative kinase activities in the presence of individual drugs compared with DMSO controls are indicated. C. The chemical structure of DSF retrieved from the database ChemSpider. D. The kinase inhibitory effects of DSF were examined at $0.001,0.01$, $0.1,0.2,1,2,10,20,30,40,50,60,70,80,90$, and $100 \mu \mathrm{M}$ in vitro. 
without excluding SNARK-independent mechanisms are implied. Noteworthily observed, on the other hand, was the suppression of SNARK mRNA levels in HepG2 cells treated by DDC (Figure 4G), which could affect SNARK-stimulated cell biological phenotypes as TGF- $\beta$ was found to upregulate SNARK expression levels (Figure $4 \mathrm{H})$, though such pronounced effects were not directly evoked by the treatment with DSF and TR (Supplementary Figure S4B and S4C). Additionally, the replication of the profibrogenic virus $\mathrm{HCV}$, dependent on SNARK [9], was also abrogated by DSF (Supplementary Figure
S4D), which was supported by a recent report using HCV replicon cells [17]. Overall, DSF's inhibition of the in vitro kinase activity results in its suppression of SNARKdependent profibrogenic signaling in HCC cells.

\section{Inhibition of SNARK-promoted HCC cell proliferation by DSF}

SNARK has been found to operate against apoptosis, functioning as a tumor promoter [18, 19]. Meanwhile, the anti-cancer effects of DSF have been
A

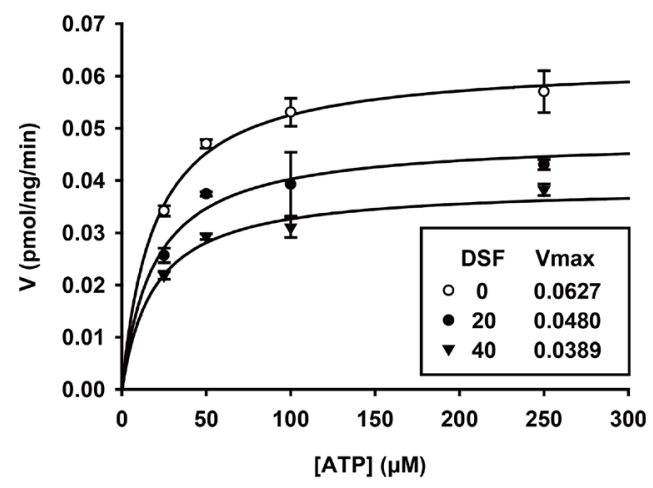

C

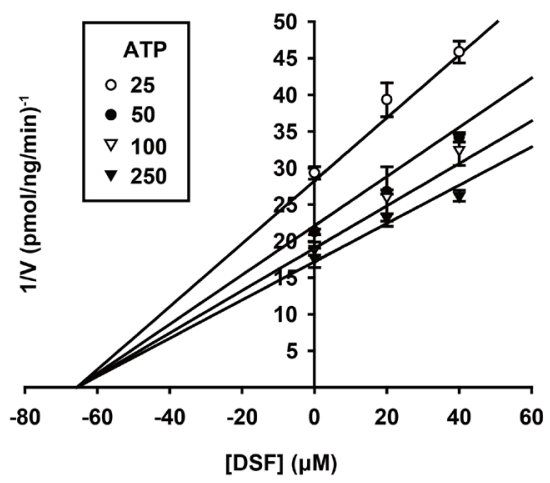

E

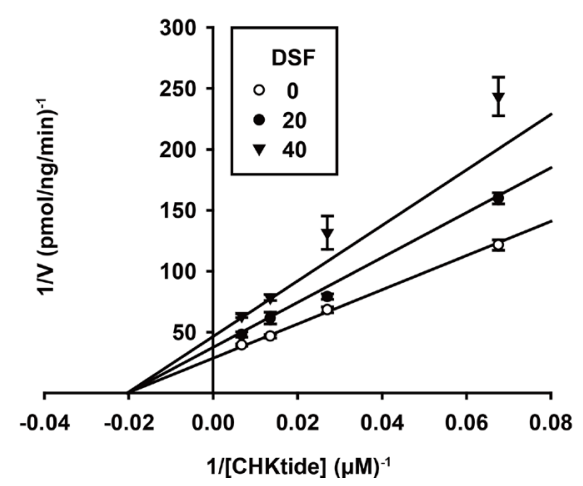

B

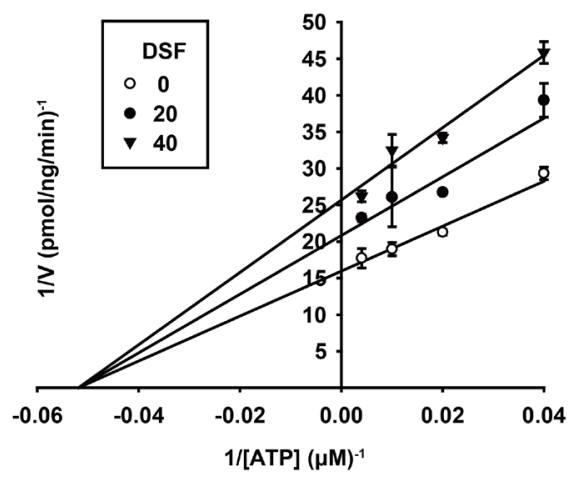

D

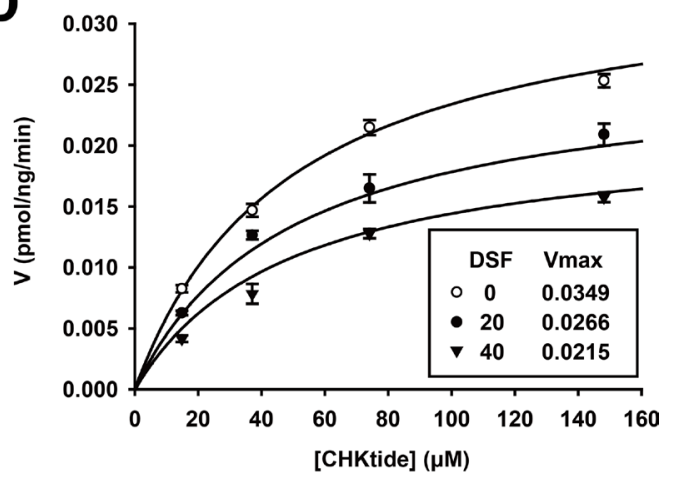

$\mathbf{F}$

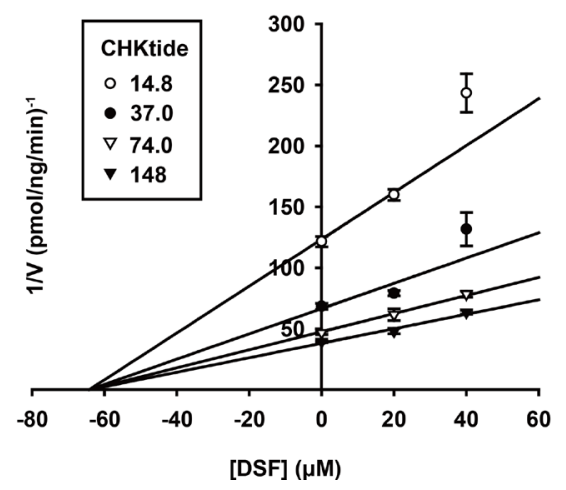

Figure 2: Enzymatic kinetics of SNARK kinase inhibition by DSF. The in vitro SNARK kinase assay was performed with 25, 50,100 , and $250 \mu \mathrm{M}$ ATP or 14.8, 37.0, 74.0, and $148 \mu \mathrm{M}$ CHKtide in the presence of 0,20 , and $40 \mu \mathrm{M}$ DSF. Subsequently effects of DSF on enzymatic kinetics of SNARK were calculated, yielding Michaelis-Menten plots A. and D. Lineweaver-Burk plots B. and E. and Dixon plots $\mathbf{C}$. and $\mathbf{F}$. 
<smiles>CN(C)C(=S)SSC(=S)N(C)C</smiles><smiles>CN(C)C(=S)SC(=S)N(C)C</smiles>

TR<smiles>CCN(CC)C(=S)[S-]</smiles>

DDC
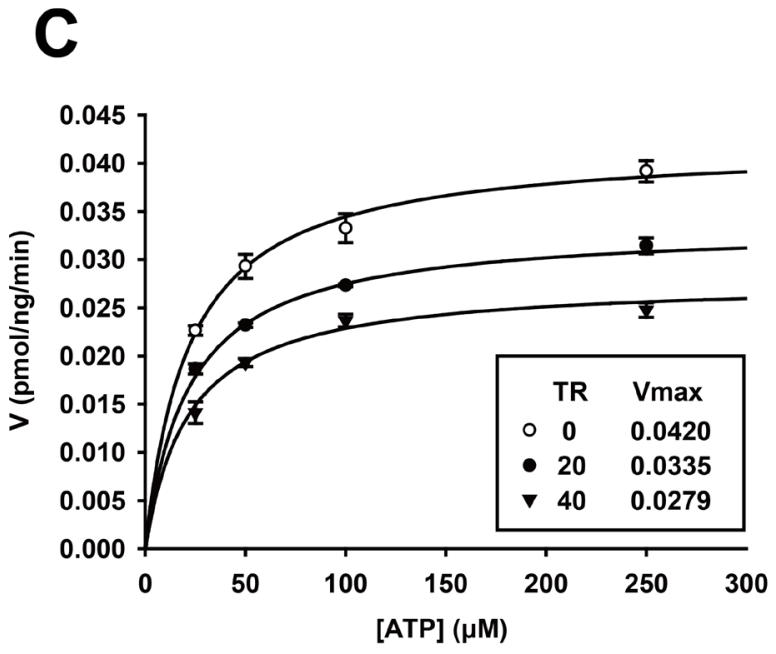

E

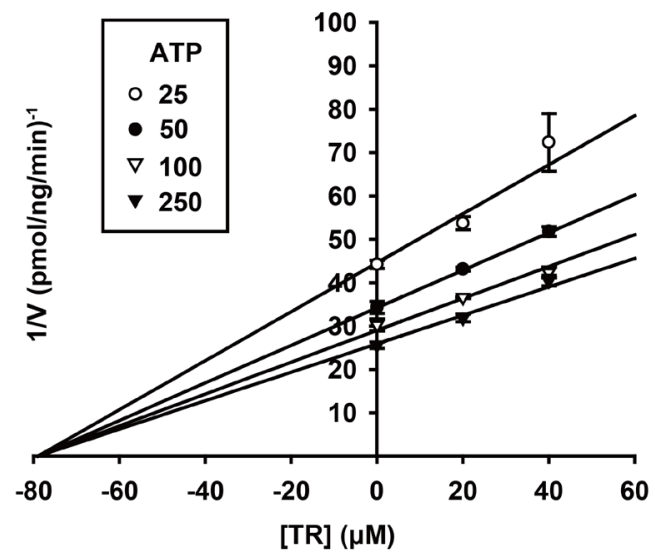

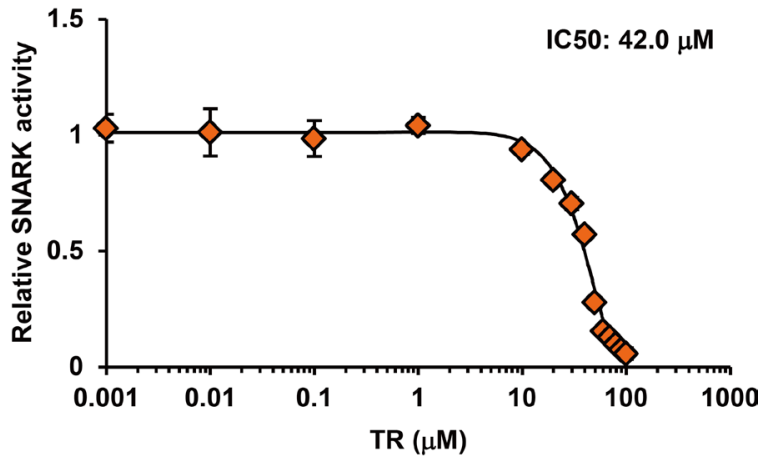

D

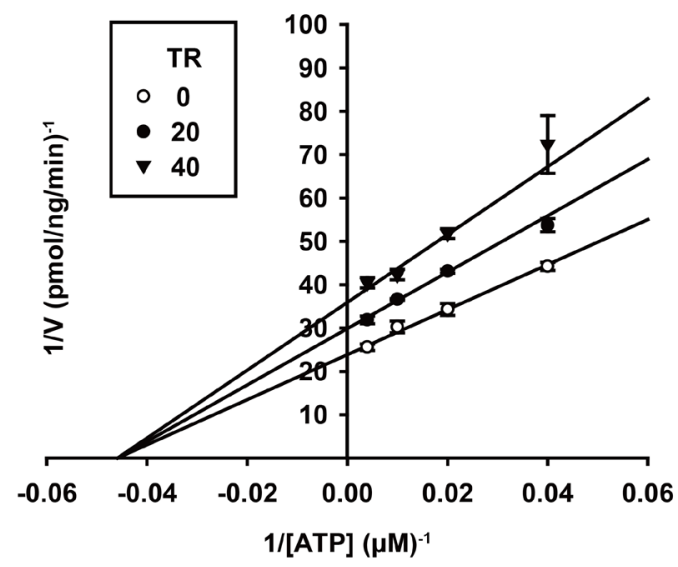

F

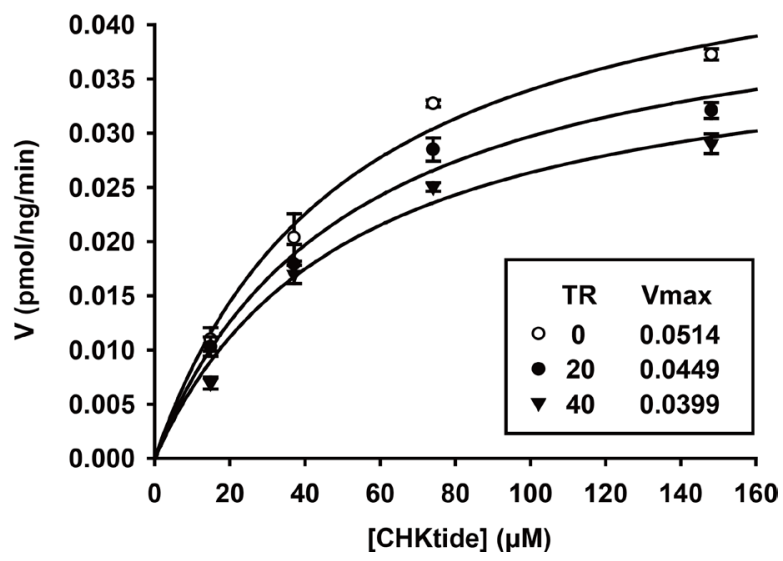

Figure 3: DSF analogs and enzymatic kinetics of SNARK kinase inhibition by TR. A. Chemical structures of TR, TMTM, and DDC were retrieved from ChemSpider. B. Similarly to Figure 1D, the kinase inhibitory effects of TR were examined at $0.001,0.01$, $0.1,1,10,20,30,40,50,60,70,80,90$, and $100 \mu \mathrm{M}$ in vitro. Similarly to Figure 2, the in vitro SNARK kinase assay was performed with $25,50,100$, and $250 \mu \mathrm{M}$ ATP or 14.8, 37.0, 74.0, and $148 \mu \mathrm{M}$ CHKtide in the presence of 0,20 , and $40 \mu \mathrm{M}$ TR. Subsequently effects of TR on enzymatic kinetics of SNARK were calculated, yielding Michaelis-Menten plots.

(Continued) 

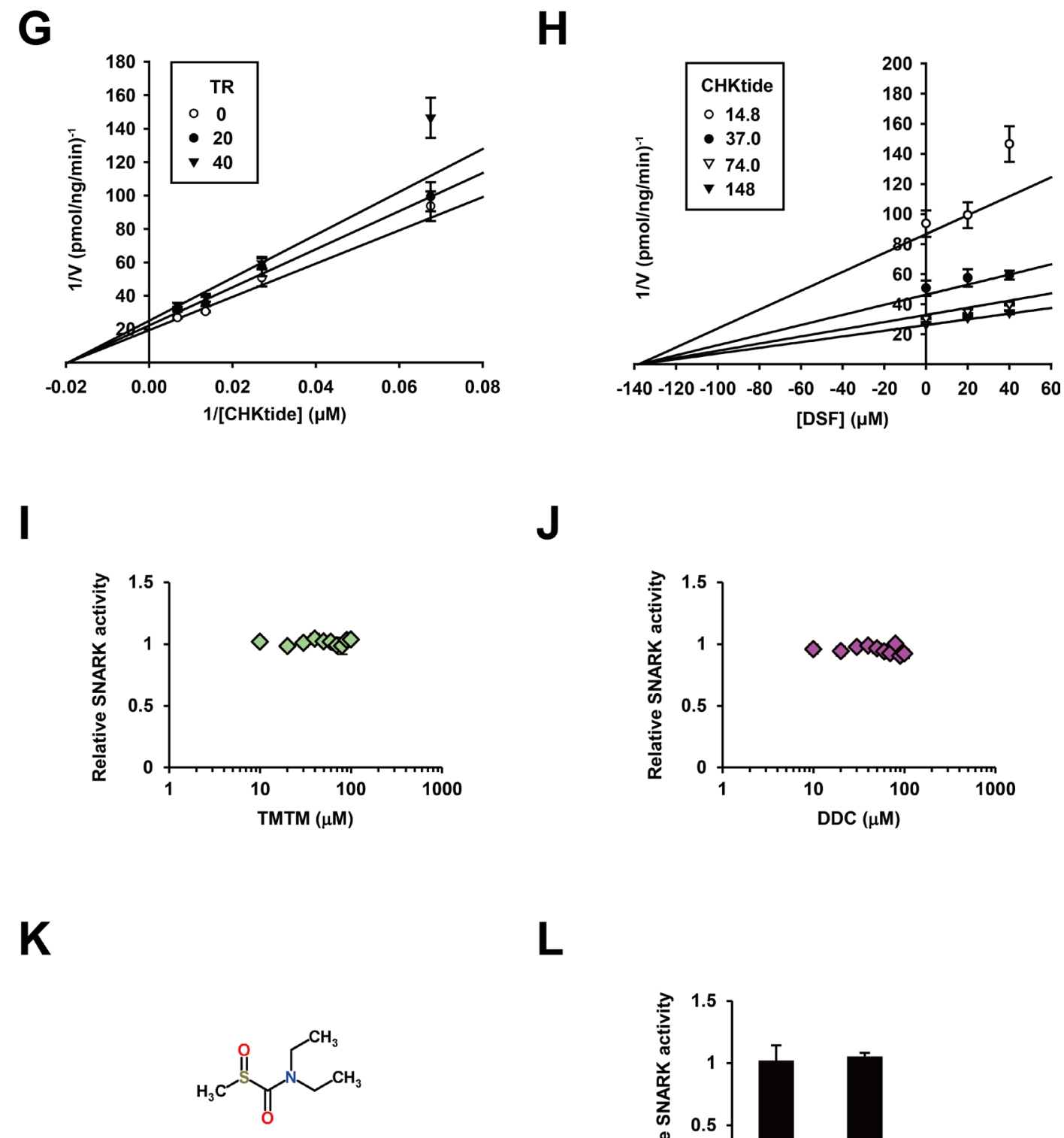

DETC-MeSO

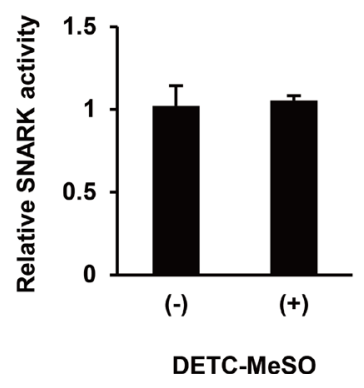

Figure 3 (Continued): C. and F. Lineweaver-Burk plots D. and G. and Dixon plots E. and H. Similarly to (B), effects of TMTM and DDC, and DETC-MeSO K. on SNARK kinase activity were examined at 10, 20, 30, 40, 50, 60, 70, 80, 90 , and $100 \mu \mathrm{M} \mathrm{I}$. and J. and 100 $\mu \mathrm{M}$ L. respectively.

described recently [12]. Hence we examined anti-HCC effects of DSF and presumed participation of SNARK in this mode of action. In HepG2 cells treated for $48 \mathrm{~h}$ (Figure 5A), DSF markedly inhibited HCC cell growth at $20 \mu \mathrm{M}$ as did TR relatively moderately, in contrast to TMTM exerting no effects at even higher concentrations, in proportion to its in vitro kinase inhibitory activity. DDC exerted pronounced anti-proliferative effects similarly to DSF though DDC itself was known to possess anti-cancer activities [20] independently. Correspondingly, remarkable cytotoxicities were caused by DSF and DDC while TR and
TMTM exerted milder and no cytotoxicities, respectively (Supplementary Figure S5A). In the other liver cancer cell line PLC/PRF/5, generally the effects of DSF and TR were stronger than those of TMTM, though DDC exhibited a unique profile (Figure 5B). These data suggest that DSF and TR demonstrate stronger cytotoxicity, particularly at higher doses (Supplementary Figure S5B), while Huh7 cells are highly susceptible to DSF (Supplementary Figure S5C), being inhibited by about $90 \%$ even at $10 \mu \mathrm{M}$, below the concentrations used for the cell-based assays in HepG2 and PLC/PRF/5 cells. Meanwhile, the levels of cell 
proliferation (Supplementary Figure S5D) and cytotoxicity (Supplementary Figure S5E) of PXB cells, normal human hepatocytes isolated from chimeric mice with a humanized liver (PXB mice) [21], were minimally altered, supporting the liver cancer cell-specific toxicity of DSF.

To determine whether SNARK was involved in the anti-HCC effects by DSF, we next conducted the assay in the presence of SNARK overexpression. Both HepG2 and PLC/PRF/5 cells were conferred resistance to DSF at lower concentrations (Figure 5C and 5D) by the excessive supply of SNARK (Supplementary Figure $\mathrm{S} 5 \mathrm{~F}$ ), which was not observed with the kinase-dead K81M and unphosphorylated T208A mutants [9] in HepG2 cells (Supplementary Figure S5G) and PLC/PRF/5 cells (Supplementary Figure S5H), confirming SNARK as a tumor promoter and a pharmacological target. Taken together, DSF exhibited anti-cancer effects against HCC as well, through its actions on SNARK.

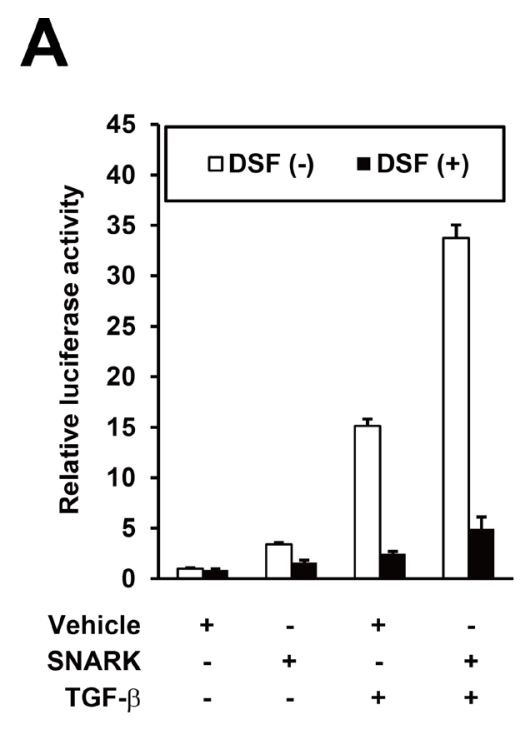

\section{B}

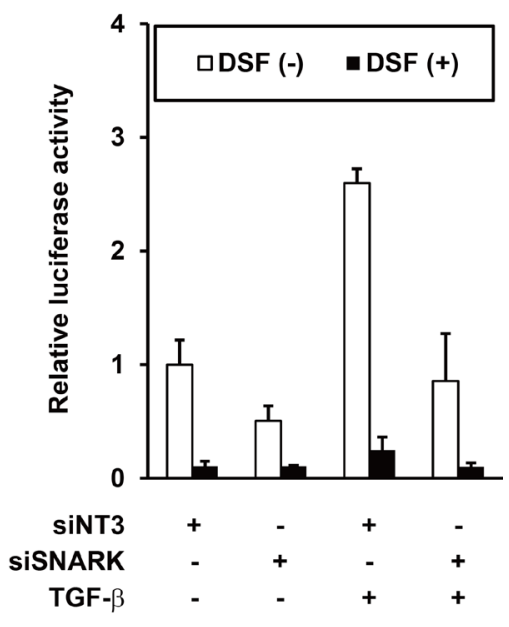

C
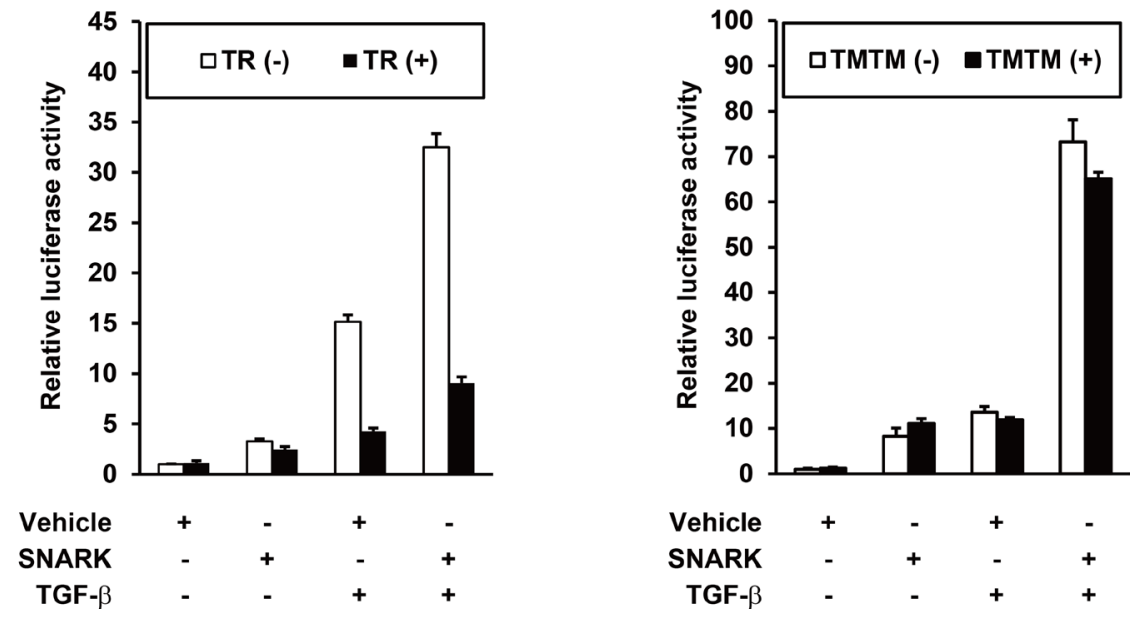

Figure 4: Inhibition of SNARK-promoted TGF- $\beta$ signaling by DSF. A. HepG2 cells were transfected with PAI/L and pRL-TK in combination with pEBMulti-Puro (Vehicle) or pEBMP-SNARK-3 $\times$ FL (SNARK), followed by the treatment with DSF at $15 \mu \mathrm{M}$ and TGF- $\beta$ at $10 \mathrm{ng} / \mathrm{mL} 24 \mathrm{~h}$ later. On the next day the cells were lysed and the firefly and Renilla luciferase activities were measured. B. HepG2 cells were transfected with non-targeting siRNAs (siNT3) or siRNAs to SNARK (siSNARK), followed by transfection with PAI/L and pRL-TK $24 \mathrm{~h}$ later. On the next day DSF at $10 \mu \mathrm{M}$ and TGF- $\beta$ at $10 \mathrm{ng} / \mathrm{mL}$ were added and $24 \mathrm{~h}$ later the cells were lysed and the firefly and Renilla luciferase activities were measured. Similarly to (A), the assays were performed using TR C. TMTM D. DDC.

(Continued) 
$\mathbf{E}$

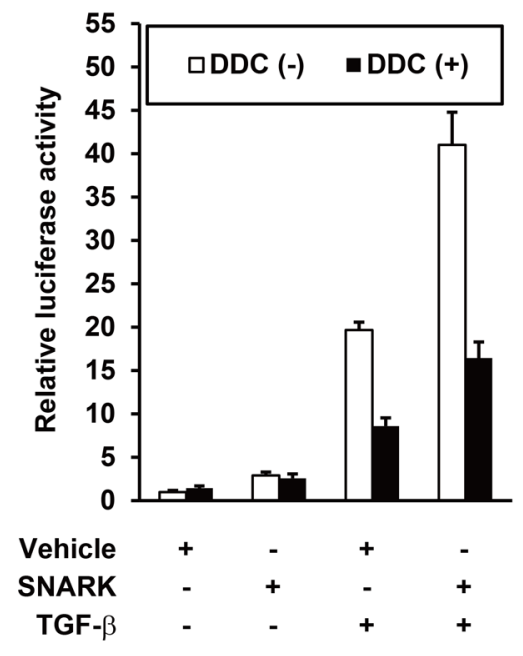

G

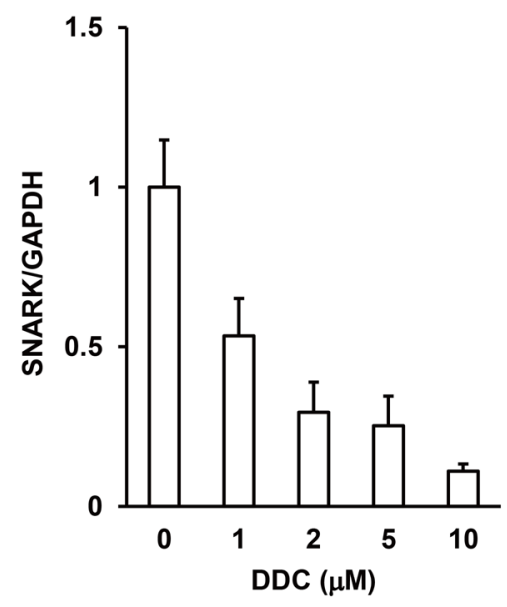

$\mathbf{F}$

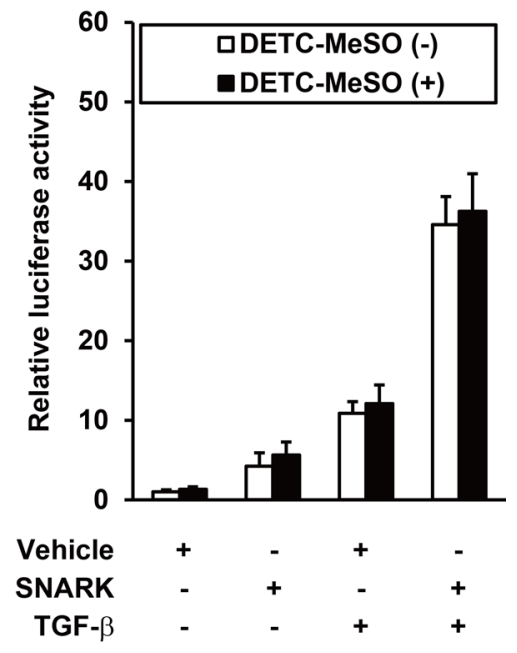

H

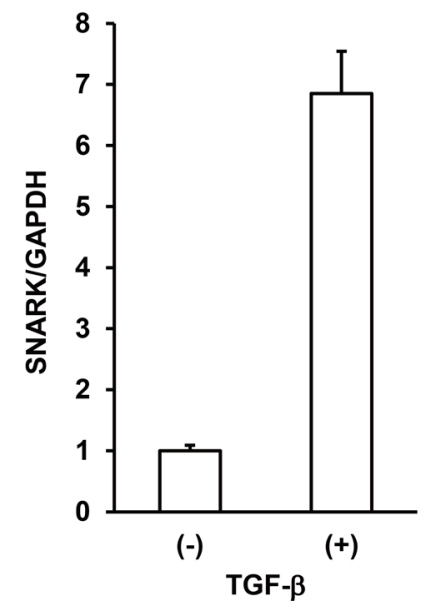

Figure 4 (Continued): E. and DETC-MeSO F. in place of DSF. Total RNA was extracted from HepG2 cells treated with DDC at indicated concentrations $\mathbf{G}$. or $10 \mathrm{ng} / \mathrm{mL}$ TGF- $\beta$ H. for $48 \mathrm{~h}$ and SNARK mRNA levels were quantified by qRT-PCR with normalization to $G A P D H . \mathrm{n} \geq 3$. Error bars $=\mathrm{SD}$.

\section{DISCUSSION}

Growing evidence has demonstrated that SNARK possesses anti-apoptotic properties $[18,19]$ and is involved in cancer [22-24]. We recently reported the novel feature of SNARK as a profibrogenic factor in HCC cells through the promotion of TGF- $\beta$ signaling [9]. Therefore the successful identification of DSF to inhibit SNARK kinase offers real opportunity to exploit the drug and dissect the mechanisms for further pharmacological development. An understanding of the basic enzymatic and biochemical properties of the SNARK-DSF interaction is important.
The enzymatic kinetics analyses suggested that DSF is an ATP-noncompetitive inhibitor (Figure 2), implying that DSF could target a regulatory site(s) for ATP hydrolysis rather than compete at the ATP-binding site. Also noncompetitively inhibited was CHKtide, connoting that a modulatory domain distinct from the catalytic center is the DSF target. It is very interesting to speculate that an identical regulatory site could affect both ATP and CHKtide reactions, though behaviors of actual proteins catalyzed in cells may not be entirely reflected by CHKtides. The identification of potential DSF-binding targets of SNARK is of great interest; the 
close DSF analog TR shared a similar 3D structure with and retained the in vitro kinase inhibitory activity in the same manner as DSF. However, TMTM, the monosulfide analog of TR, was conformationally distinct from DSF and TR and devoid of the kinase inhibition. The disulfide bond-based structure is assumed to be fit for DSF docking with the kinase, and accordingly further structural analyses including crystallography of SNARK and its complex with DSF are warranted based on the insights from DSF and its analogs as chemical probes. This could stimulate rational drug development through the well-known characteristic of DSF.

In our assays, SNARK was shown to promote TGF- $\beta$ signaling in HCC cells, which was suppressed by the SNARK inhibitor DSF as expected. Intriguingly, a recent study reported that DSF inhibited TGF- $\beta$-induced epithelial-mesenchymal transition in breast cancer cells [11] in conformity with the downregulation of TGF- $\beta$ signaling by DSF in zebra fish cells [25]. Furthermore, fibrosis-attenuating properties of DSF were observed in a mouse model of non-alcoholic steatohepatitis [26]. Therefore SNARK has been projected as the target of anti-fibrogenic effects through TGF- $\beta$ signaling by DSF. As for DDC, a DSF metabolite, though it lacks SNARK kinase inhibition in vitro, suppressive effects on PAI/L when treated with TGF- $\beta$ were still detected, potentially indicating intracellular circumstance-specific suppression of TGF- $\beta$ signaling. Of interest, DDC, but not DSF (Supplementary Figure S4B), was found to decrease the SNARK expression levels (Figure 4G), which was in actuality elevated by TGF- $\beta$ (Figure $4 \mathrm{H}$ ). This could at least partly explain the retained suppressive effects of DDC particularly in the presence of TGF- $\beta$, and denoted the effectiveness of breaking the vicious circle of reciprocal upregulation between SNARK and TGF- $\beta$. The enzymatic and expression inhibition of SNARK by DSF and its metabolite DDC, respectively, are thus postulated to accentuate the potency of DSF coordinately, and further detailed mechanistic studies are underway to decipher these findings.

The noted function of SNARK as a tumor promoter with anti-apoptotic properties was indeed verified in HCC cells through observation of their resistance to the cytotoxic effects of DSF conferred by the excessive supply of SNARK. In recent years, mounting evidence suggests anti-cancer properties of DSF across cancer types [12], and HCC cells are no exception; DSF exerted anti-HCC effects through inhibition of hypoxia-induced

B

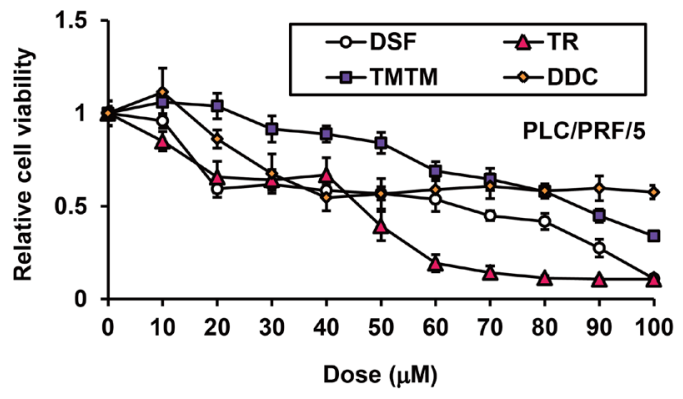

D

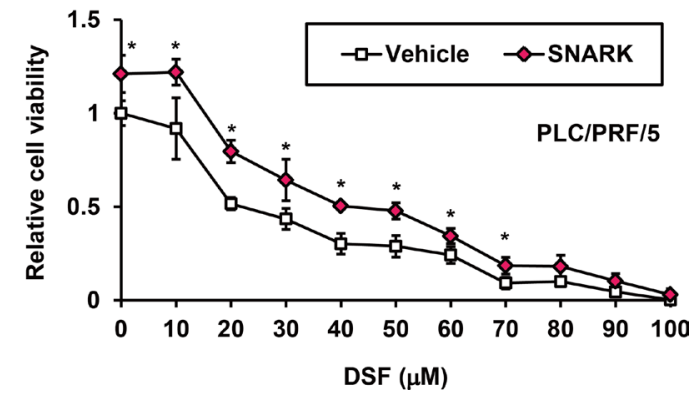

Figure 5: Inhibition of SNARK-promoted HCC cell proliferation by DSF. HepG2 A. and PLC/PRF/5 B. cells were treated with DSF, TR, TMTM, and DDC at the indicated doses for 24 hours and the relative cell viabilities to untreated control cells were measured by CCK-8 assay. pEBMulti-Puro (Vehicle) or pEBMP-SNARK-3×FL (SNARK) was transfected into HepG2 C. or PLC/PRF/5 D. cells, followed by DSF treatment at the indicated concentrations $48 \mathrm{~h}$ later. On the next day the relative cell viabilities to the control samples untreated and transfected with pEBMulti-Puro were measured similarly to (A) and (B). ${ }^{*} P<0.05$ by Student's t-test. $\mathrm{n}=4$. Error bars $=\mathrm{SD}$. 
gene expression and hypoxia-inducible factor activity for tumor adaptation to hypoxia [27]. Also tumorigenicity of tumor-initiating HCC cells was impaired via reactive oxygen spieces-p38 pathway and Glypican 3 by DSF [28]. Another recent study showed DSF, in Hep3B cells, triggered apoptosis intrinsically and extrinsically, including activation of the pathway through death receptor CD95 [29], through which SNARK reportedly rendered breast cancer cells apoptosis-resistant and invasive [18]. Such modes of anti-cancer actions by DSF as above allude to critical molecules associated with and potentially phosphorylated as substrates by SNARK, whose unknown functions attract much attention for reciprocal elucidation between physiological roles and therapeutic implications. Again DDC, with anti-cancer effects via chelating into metal ions [20] and reported to significantly improve the overall survival in breast cancer patients [30], demonstrated unique profiles of cytotoxicity to HCC cells, and indicated the effects by DSF are to be kept even after being metabolized at least reducing SNARK expression (Figure 4G); lately further improvement in stable delivery and anti-cancer effects of DDC was reported in vivo [31]. Utilization of intact DSF, simultaneously, is being investigated for its anti-cancer properties, and such a method was recently developed by nanocapsule-protected medicine [32], achieving favorable anti-cancer effects. Still the cytotoxic efficacy could fluctuate depending on individual cell types with various genetic and pathoetiological backgrounds as was the case with PLC/PRF/5 cells positive for hepatitis B virus proteins (Figure 4B) and anti-cancer agents [33]. Thus, together with the observation of suppressive effects on TGF- $\beta$ signaling, leading to the inhibition of cancer cell metastasis and tumor growth in vivo [11], DSF is thought to be an effective therapeutic agent for solid tumors including $\mathrm{HCC}$.

In practice, therapeutic effects of DSF have been observed in metastatic non-small cell lung cancer (NSCLC) patients with an increase in survival [34] and solid tumors [35], and also is under clinical trials for glioblastoma [12, 36] and advanced HCC (UMIN000008529, http://www.umin.ac.jp/) in expectation of exploitation as a chemotherapeutic agent. Another attractive feature of DSF is the tolerability and safety demonstrated over years of clinical experience with a large number of patients, and also in the NSCLC patients [34]. Plasma concentrations of DSF in several reports [37, 38], reaching even above $100 \mu \mathrm{M}$, routinely exceeded the $\mathrm{IC}_{50}$ of the in vitro kinase assay described here, so SNARK inhibitory concentrations are expected to be achieved safely in patients. It should be cautioned that the possible novel clinical use of DSF would be in persons with chronic liver disease at risk for cirrhosis and HCC, and understandably toxicity would need to be carefully monitored especially in persons with advanced cirrhosis, as summarized in disease guidelines [39]. However, DSF has long been used safely in patients who often harbor significant alcoholic liver disease.

Here the enzymatic inhibition of SNARK and resultant anti-TGF- $\beta / \mathrm{HCC}$ effects by DSF provide us with new biochemical and pragmatic insights. The utility and a newly revealed property of DSF were mechanistically suggested and in turn the significance of regulatory sites for SNARK kinase activity as validated drug target can now be comprehensively assessed using DSF as a chemical probe. Also DSF derivatives with better potencies could be examined [40] in addition to devising improved methods of delivery $[32,41]$. Further chemical and biological analyses would accelerate understanding of SNARK functions and at the same time the chemical search for and development of potent SNARK inhibitors continue. Finally well-tolerated and more effective regimens for HCC management can be expected to be developed using DSF and its analogs/derivatives, inspired and reinforced by the discovery in this study.

\section{MATERIALS AND METHODS}

\section{Compounds and cells}

DSF, and STS, TR, TMTM and Sodium diethyldithiocarbamate trihydrate were purchased from Selleckchem (Houston, TX) and Sigma-Aldrich (St. Louis, MO), respectively. DETC-MeSO was purchased from Santa Cruz Biotechnology (Santa Cruz, CA). TGF- $\beta$ was purchased from R\&D (Minneapolis, MN). Antibodies to luciferase, HCV NS3, and FLAG and $\beta$-actin (ACTB) were purchased from MBL (Aichi, Japan), Abcam (Cambridge, United Kingdom), and Sigma-Aldrich, respectively. The FDA-Approved Drug Screen-well Library and Cell Counting Kit (CCK)-8 were obtained from Enzo Life Sciences (Farmingdale, NY) and Dojindo (Kumamoto, Japan), respectively. Cytotoxicities of compounds were determined with the LDH cytotoxicity detection kit (Takara Bio, Shiga, Japan). HepG2, PLC/PRF/5, and Huh7 cells were cultured according to the protocols of American Type Culture Collection (Manassas, VA) and Japanese Collection of Research Bioresources Cell Bank (Osaka, Japan). The cell lines were authenticated by short tandem repeat method (Bex, Tokyo, Japan) in Jan 2016. PXB cells were purchased from Phoenix Bio (Hiroshima, Japan). JFH1 virus infection was performed as described previously [9].

\section{Plasmid}

pCMV14-SNARK_NCBI-3×FL was constructed by primers EcoRI-SNARK-F 5'-TTGAATTCGGCCA CCATGGAGTCGCTGGTTTTC-3', XbaI-SNARK- $\Delta$ T GA-R 5'-AATTCTAGAGGTGAGCTTTGAGCAGAC -3', SNARK-1323-G-F 5'-GGCAGGCTGCCCCGCTG CTC-3', and SNARK-1323-G-R 5'-GAGCAGCGGGGCA 
GCCTGCC-3', using pCMV14-SNARK-3×FL as a template [9]. Sequences containing SNARK open reading frame were amplified by the primers XhoI- SNARK-F 5'-ATTC TCGAGGGCCACCATGGAGTCGCTGGTTTTC-3' and NotI-SNARK-3×FL-R 5'-AATGCGGCCGCCTACTTGT CATCGTCATCCTTG-3' from pCMV14- SNARK_NCBI$3 \times \mathrm{FL}$, and subcloned into pEBMulti-Puro (Wako, Osaka, Japan), producing the expression plasmid pEBMP-SNARK$3 \times$ FL. Similarly, pCMV14-SNARK(K81M)_NCBI$3 \times$ FL and pCMV14-SNARK(T208A)_NCBI-3 $\times$ FL were generated by primers EcoRI-SNARK-F, XbaI-SNARK$\triangle$ TGA-R, SNARK-1323-G-F and SNARK-1323-G-R using pCMV14-SNARK(K81M)-3×FL and pCMV14SNARK(T208A)-3×FL as templates, respectively [9]; subsequently using these constructs as templates, pEBMPSNARK(K81M)-3×FL and pEBMP-SNARK(T208A)$3 \times$ FL were produced by primers EcoRI-SNARK-F and NotI-SNARK-3×FL-R. pEBMP-Luc was constructed by primers XhoI-Luc-F 5'-ATTCTCGAGGGCCACCATGG AAGACGCCAAAAACATAAAGAAAGGC-3' and NotILuc-R AATGCGGCCGCTTACAATTTGGACTTTCCGC CCTTCTTGGC using PAI/L as a template.

\section{In vitro kinase assay}

NUAK2 Kinase Enzyme System was purchased from Promega (Madison, WI). Kinase assay was performed according to the manufacturer's protocol and the luminescence was monitored $(n=4)$. Briefly, $12.5 \mathrm{ng}$ of recombinant SNARK was incubated with CHKtide and ATP, in the presence or absence of DMSO or compounds, for 60 min at room temperature, followed by ATP depletion and subsequent detection of converted ADP via luminescence.

\section{Enzyme kinetic analysis}

The mode of enzymatic inhibition was calculated bySigmaplot (Systat Software, San Jose, CA), with the best fit models determined by the Akaike Information Criterion.

\section{Luciferase assay}

Firefly luciferase activity was monitored by a dualluciferase reporter assay system (Promega) and normalized to Renilla luciferase activities from pRL-TK as described previously [9]. SiNT3 (\#D-001210-03) and siSNARK (SI02660224) were purchased from GE Healthcare BioSciences (Pittsburgh, PA) and QIAGEN (Valencia, CA), respectively, and the assay was performed as described previously [9], with 3 pmol siRNAs at $5 \mathrm{nM}$.

\section{Quantitative reverse transcription-polymerase chain reaction}

Relative mRNA and viral RNA levels were quantified as previously described [9] using the following primer sets:
SNARK-F 5'-GATGCACATACGGAGGGAGAT-3' and SNARK-R 5'-GCTGGCATACTCCATGACGAT-3' for SNARK, JFH1-F 5'-CTGTCTTCACGCAGAAAGCG-3' and JFH1-R 5'- TCGCAACCCAACGCTACTCG-3' and GAPDH-F2 5'-AAGGTGAAGGTCGGAGTCAAC-3' and GAPDH-R2 5'-GGGGTCATTGATGGCAACAATA-3' for glyceraldehyde-3-phosphate dehydrogenase (GAPDH), with the value of SNARK and JFH1 normalized to that of GAPDH.

\section{Western blotting}

Total protein was resolved by SDS-PAGE and subjected to western blotting as described previously [9].

\section{ACKNOWLEDGMENTS}

We thank Dr. Soichi Kojima for PAI/L reporter.

\section{CONFLICTS OF INTEREST}

The authors disclose no conflicts of interest.

\section{GRANT SUPPORT}

N.K. is supported by Grants-in-Aid for Scientific Research from the Ministry of Education, Culture, Sports, Science and Technology in Japan (24390184) and Japan Agency for Medical Research and Development (15fk0310009h0004). K.G. is supported by a Grant-in-Aid for Young Scientist (15K19106) from the Japan Society for the Promotion of Science.

\section{REFERENCES}

1. Cohen P, Alessi DR. Kinase drug discovery--what's next in the field? ACS Chem Biol. 2013; 8:96-104.

2. Roskoski R, Jr. A historical overview of protein kinases and their targeted small molecule inhibitors. Pharmacol Res. 2015; 100:1-23.

3. Novikova DS, Garabadzhiu AV, Melino G, Barlev NA, Tribulovich VG. AMP- activated protein kinase: structure, function, and role in pathological processes. Biochemistry (Mosc). 2015; 80:127-144.

4. Monteverde T, Muthalagu N, Port J, Murphy DJ. Evidence of cancer-promoting roles for AMPK and related kinases. FEBS J. 2015; 282:4658-4671.

5. Goto K, Kato N. MICA SNPs and the NKG2D system in virus-induced HCC. J Gastroenterol. 2015; 50:261-272.

6. Giannelli G, Mikulits W, Dooley S, Fabregat I, Moustakas A, Ten Dijke P, Portincasa P, Winter P, Janssen R, Leporatti S, Herrera B, Sanchez A. The Rationale for Targeting TGFbeta in Chronic Liver Diseases. Eur J Clin Invest. 2016.

7. Sun X, Gao L, Chien HY, Li WC, Zhao J. The regulation and function of the NUAK family. J Mol Endocrinol. 2013; 51:R15-22. 
8. Tai AW, Benita Y, Peng LF, Kim SS, Sakamoto N, Xavier RJ, Chung RT. A functional genomic screen identifies cellular cofactors of hepatitis $\mathrm{C}$ virus replication. Cell Host Microbe. 2009; 5:298-307.

9. Goto K, Lin W, Zhang L, Jilg N, Shao RX, Schaefer EA, Zhao H, Fusco DN, Peng LF, Kato N, Chung RT. The AMPK-related kinase SNARK regulates hepatitis C virus replication and pathogenesis through enhancement of TGFbeta signaling. J Hepatol. 2013; 59:942-948.

10. Ashburn TT, Thor KB. Drug repositioning: identifying and developing new uses for existing drugs. Nat Rev Drug Discov. 2004; 3:673-683.

11. Han D, Wu G, Chang C, Zhu F, Xiao Y, Li Q, Zhang T, Zhang L. Disulfiram inhibits TGF-beta-induced epithelialmesenchymal transition and stem-like features in breast cancer via ERK/NF-kappaB/Snail pathway. Oncotarget. 2015; 6:40907-40919. doi: 10.18632/oncotarget.5723.

12. Triscott J, Rose Pambid M, Dunn SE. Concise review: bullseye: targeting cancer stem cells to improve the treatment of gliomas by repurposing disulfiram. Stem Cells. 2015; 33:1042-1046.

13. Akbar M, Kim HY. Protective effects of docosahexaenoic acid in staurosporine- induced apoptosis: involvement of phosphatidylinositol-3 kinase pathway. J Neurochem. 2002; 82:655-665.

14. Auld DS, Southall NT, Jadhav A, Johnson RL, Diller DJ, Simeonov A, Austin CP, Inglese J. Characterization of chemical libraries for luciferase inhibitory activity. J Med Chem. 2008; 51:2372-2386.

15. Koppaka V, Thompson DC, Chen Y, Ellermann M, Nicolaou KC, Juvonen RO, Petersen D, Deitrich RA, Hurley TD, Vasiliou V. Aldehyde dehydrogenase inhibitors: a comprehensive review of the pharmacology, mechanism of action, substrate specificity, and clinical application. Pharmacol Rev. 2012; 64:520-539.

16. Abe M, Harpel JG, Metz CN, Nunes I, Loskutoff DJ, Rifkin DB. An assay for transforming growth factor-beta using cells transfected with a plasminogen activator inhibitor-1 promoter-luciferase construct. Anal Biochem. 1994; 216:276-284.

17. Lee YM, Duh Y, Wang ST, Lai MM, Yuan HS, Lim C. Using an Old Drug to Target a New Drug Site: Application of Disulfiram to Target the Zn-Site in HCV NS5A Protein. J Am Chem Soc. 2016; 138:3856-3862.

18. Legembre P, Schickel R, Barnhart BC, Peter ME. Identification of SNF1/AMP kinase-related kinase as an NF-kappaB-regulated anti-apoptotic kinase involved in CD95-induced motility and invasiveness. J Biol Chem. 2004; 279:46742-46747.

19. Lessard SJ, Rivas DA, So K, Koh HJ, Queiroz AL, Hirshman MF, Fielding RA, Goodyear LJ. The AMPKrelated kinase SNARK regulates muscle mass and myocyte survival. J Clin Invest. 2016.
20. Cen D, Brayton D, Shahandeh B, Meyskens FL, Jr., Farmer PJ. Disulfiram facilitates intracellular $\mathrm{Cu}$ uptake and induces apoptosis in human melanoma cells. J Med Chem. 2004; 47:6914-6920.

21. Kakuni M, Yamasaki C, Tachibana A, Yoshizane Y, Ishida Y, Tateno C. Chimeric mice with humanized livers: a unique tool for in vivo and in vitro enzyme induction studies. Int $\mathrm{J}$ Mol Sci. 2014; 15:58-74.

22. Kim JH, Kim WS, Park C. SNARK, a novel downstream molecule of EBV latent membrane protein 1, is associated with resistance to cancer cell death. Leuk Lymphoma. 2008; 49:1392-1398.

23. Namiki T, Tanemura A, Valencia JC, Coelho SG, Passeron T, Kawaguchi M, Vieira WD, Ishikawa M, Nishijima W, Izumo T, Kaneko Y, Katayama I, Yamaguchi Y, Yin L, Polley EC, Liu H, et al. AMP kinase-related kinase NUAK2 affects tumor growth, migration, and clinical outcome of human melanoma. Proc Natl Acad Sci U S A. 2011; 108:6597-6602.

24. Namiki T, Yaguchi T, Nakamura K, Valencia JC, Coelho SG, Yin L, Kawaguchi M, Vieira WD, Kaneko Y, Tanemura A, Katayama I, Yokozeki H, Kawakami Y, Hearing VJ. NUAK2 Amplification Coupled with PTEN Deficiency Promotes Melanoma Development via CDK Activation. Cancer Res. 2015; 75:2708-2715.

25. van Boxtel AL, Pieterse B, Cenijn P, Kamstra JH, Brouwer A, van Wieringen W, de Boer J, Legler J. Dithiocarbamates induce craniofacial abnormalities and downregulate sox9a during zebrafish development. Toxicol Sci. 2010; 117:209-217.

26. Schwartz JJ, Emerson L, Hillas E, Phan A, Thiesset H, Firpo M, Sorensen J, Kennedy T, Rinella M. Amelioration of hepatic inflammation in a mouse model of NASH using a dithiocarbamate derivative. Hepatol Int. 2013; 7:600-609.

27. Park HJ, Kim MS, Cho K, Yun JH, Choi YJ, Cho CH. Disulfiram deregulates HIF- alpha subunits and blunts tumor adaptation to hypoxia in hepatoma cells. Acta Pharmacol Sin. 2013; 34:1208-1216.

28. Chiba T, Suzuki E, Yuki K, Zen Y, Oshima M, Miyagi S, Saraya A, Koide S, Motoyama T, Ogasawara S, Ooka Y, Tawada A, Nakatsura T, Hayashi T, Yamashita T, Kaneko S, et al. Disulfiram eradicates tumor-initiating hepatocellular carcinoma cells in ROS-p38 MAPK pathway-dependent and -independent manners. PLoS One. 2014; 9:e84807.

29. Hoda M, Pajaniradje S, Shakya G, Mohankumar K, Rajagopalan R. Anti-proliferative and apoptosis-triggering potential of disulfiram and disulfiram-loaded polysorbate 80- stabilized PLGA nanoparticles on hepatocellular carcinoma Hep3B cell line. Nanomedicine. 2016.

30. Dufour P, Lang JM, Giron C, Duclos B, Haehnel P, Jaeck D, Jung JM, Oberling F. Sodium dithiocarb as adjuvant immunotherapy for high risk breast cancer: a randomized study. Biotherapy. 1993; 6:9-12. 
31. Wehbe M, Anantha M, Backstrom I, Leung A, Chen K, Malhotra A, Edwards K, Bally MB. Nanoscale Reaction Vessels Designed for Synthesis of Copper-Drug Complexes Suitable for Preclinical Development. PLoS One. 2016; 11:e0153416.

32. Zhang L, Tian B, Li Y, Lei T, Meng J, Yang L, Zhang Y, Chen F, Zhang H, Xu H, Tang X. A Copper-Mediated Disulfiram-Loaded $\mathrm{pH}$-Triggered PEG-Shedding TAT Peptide-Modified Lipid Nanocapsules for Use in Tumor Therapy. ACS Appl Mater Interfaces. 2015; 7:25147-25161.

33. Pinyol R, Nault JC, Quetglas IM, Zucman-Rossi J, Llovet $\mathrm{JM}$. Molecular profiling of liver tumors: classification and clinical translation for decision making. Semin Liver Dis. 2014; 34:363-375.

34. Nechushtan H, Hamamreh Y, Nidal S, Gotfried M, Baron A, Shalev YI, Nisman B, Peretz T, Peylan-Ramu N. A phase IIb trial assessing the addition of disulfiram to chemotherapy for the treatment of metastatic non-small cell lung cancer. Oncologist. 2015; 20:366-367.

35. Cvek B. Nonprofit drugs as the salvation of the world's healthcare systems: the case of Antabuse (disulfiram). Drug Discov Today. 2011; 17:409-412.

36. Huang J, Campian JL, Gujar AD, Tran DD, Lockhart AC, DeWees TA, Tsien CI, Kim AH. A phase I study to repurpose disulfiram in combination with temozolomide to treat newly diagnosed glioblastoma after chemoradiotherapy. J Neurooncol. 2016.

37. Malcolm MT. Disulfiram blood levels. Br Med J. 1977; 2:457.

38. Cid E, Cid E, Jr., Feuerhake O, Boisier I, Chandia R. Plasma concentrations of disulfiram after injection of suspended micropellets into alcoholic subjects. Biopharm Drug Dispos. 1991; 12:163-169.

39. Center for Substance Abuse Treatment. Incorporating Alcohol Pharmacotherapies Into Medical Practice: A Review of the Literature. Rockville (MD): Substance Abuse and Mental Health Services Administration (US); 2009. (Treatment Improvement Protocol (TIP) Series, No. 49s.).

40. Sharma V, Verma V, Lal N, Yadav SK, Sarkar S, Mandalapu D, Porwal K, Rawat T, Maikhuri JP, Rajender S, Sharma VL, Gupta G. Disulfiram and its novel derivative sensitize prostate cancer cells to the growth regulatory mechanisms of the cell by re- expressing the epigenetically repressed tumor suppressor-estrogen receptor beta. Mol Carcinog. 2015.

41. Fasehee H, Zarrinrad G, Tavangar SM, Ghaffari SH, Faghihi $\mathrm{S}$. The inhibitory effect of disulfiram encapsulated PLGA NPs on tumor growth: Different administration routes. Mater Sci Eng C Mater Biol Appl. 2016; 63:587-595. 